\title{
A Deep Large Scale Survey for Intermediate Age Brown Dwarfs in the Praesepe Cluster
}

\author{
R. J. Chappelle ${ }^{1}$ D. J. Pinfield, I. A. Steele \\ Astrophysics Research Institute, Liverpool John Moores University, \\ Egerton Wharf, Birkenhead CH41 1LD, UK
}

\begin{abstract}
We present a 1.4 square degree survey of the Praesepe open cluster complete to $\mathrm{I}=21.3$ and $\mathrm{Z}=20.2$. This corresponds to masses down to $0.06 M_{\odot}$ and $0.075 M_{\odot}$ for an assumed cluster age of 1.0 or 0.5 Gyrs respectively. Over fifty new Praesepe candidate members have been discovered with masses less than $0.25 M_{\odot}$. We discuss plans to establish proper motions of identified Praesepe candidates, and to measure NIR photometry of our candidates.
\end{abstract}

\section{Introduction}

Praesepe (M44) is an open cluster in Cancer, at a distance of some 160 parsecs (distance modulus $\approx 6.0$ ), with an intermediate age from $0.5-1.0$ Gyr. It is compact on the sky, and suffers low reddening and extinction (Crawford 1969). These qualities make it an ideal cluster in which to identify intermediate age BDs. Several studies have been published on Praesepe probing to low stellar masses (Hambly et al. 1995, hereafter HSHJ; Pinfield et al. 1997). In this paper, we present some of the first results of a new deeper I and Z band CCD survey of Praesepe.

\section{Observations}

We obtained I and $\mathrm{Z}$ band images for 1.4 square degrees of Praesepe taken with the WFC instrument on the Isaac Newton Telescope, La Palma, during December 2001. Conditions were photometric for 2 of the 5 nights. We have so far extracted photometry from the five fields taken on these nights using standard IRAF routines. In addition, we have recently acquired 2 extra fields in INT service time, as well as calibration images for the remainder of our survey.

\footnotetext{
${ }^{1}$ e-mail address rjc@astro.livjm.ac.uk
} 

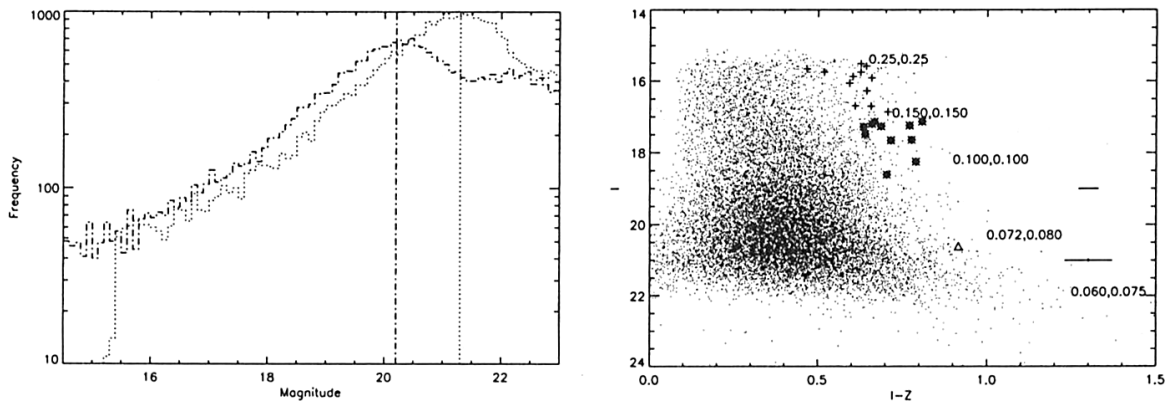

Figure 1. Source counts against magnitude plot and colour magnitude diagram of our survey. HSHJ candidates (crosses), Pinfield candidates(squares) and Roque PR1 (triangle) are highlighted. Mass estimates are from the $0.5 \mathrm{Gyr}$ and $1.0 \mathrm{Gyr}$ NextGen isochrones. (I and $\mathrm{Z}$ band completeness limits (dotted and dashed respectively) are indicated on the first plot).

\section{Results \& Discussion}

The source counts against magnitude plot and the I,I-Z colour magnitude diagram for the survey (calibrated so far) are shown in figure 1 . The survey is complete to $I=21.3$ and $Z=20.2$, going further into the low-mass regime than previous studies. We have identified 14 candidates from the HSHJ survey and 11 candidates from the Pinfield survey, as well as Roque Pr1 (Magazzù et al. 1998). Based on the location of these previously known members, we have identified around fifty new low-mass candidate members with masses between $0.25 M_{\odot}$ and $0.06 M_{\odot}$. More than half of these candidates are potential BDs.

We are currently measuring proper motions for previously known candidates, by combining our new image data with that from 1995 and 1996. In the future we plan to obtain follow-up JHK photometry of our new candidates. This data will provide cluster membership criteria, and allow us to fit the age of the cluster from the position of the BD sequence in the I, I-J colour magnitude diagram. We will also identify unresolved binaries, and derive a mass function using theoretical BD masses. By comparing the Praesepe BD population to that of younger clusters like the Pleiades, we plan to investigate cluster evolution and the nature of $\mathrm{BD}$ binaries.

\section{References}

Crawford D. L., 1969, AJ, 74, 818

Hambly, N.C. et al., 1995, MNRAS, 273, 505

Hodgkin, S. T. et al., 1999, MNRAS, 310, 87

Jones, B. F., \& Stauffer, J. R. 1991, AJ, 102, 1080

Magazzù, A. et al., 1998, ApJ, 497, L47

Pinfield, D. J. et al., 1997, MNRAS, 287, 180 